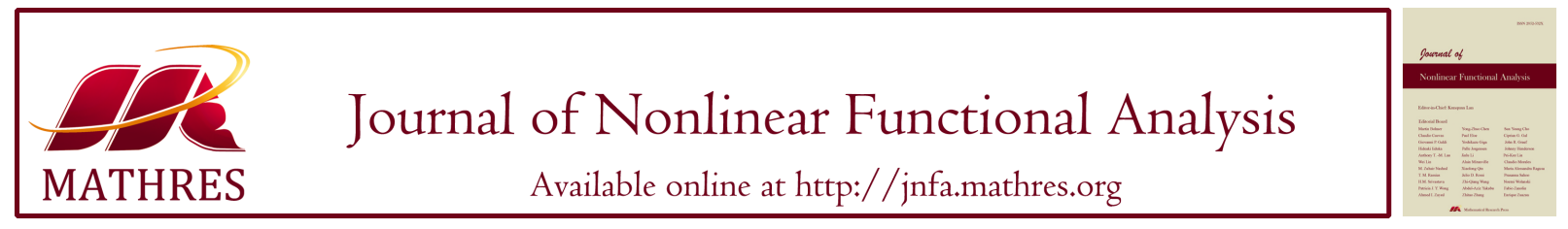

\title{
ZERO-HOPF BIFURCATIONS AND CHAOS OF QUADRATIC JERK SYSTEMS
}

\author{
BO SANG ${ }^{1, *}$, RIZGAR SALIH ${ }^{2}$, NING WANG ${ }^{3}$ \\ ${ }^{1}$ School of Mathematical Sciences, Liaocheng University, Liaocheng 252059, China \\ ${ }^{2}$ College of Basic Education, University of Raparin, Kurdistan Region, Iraq \\ ${ }^{3}$ School of Electrical and Information Engineering, Tianjin University, Tianjin 300072, China
}

\begin{abstract}
The purpose of this paper is to propose some coefficient conditions, characterize the stability of the periodic solutions bifurcated from zero-Hopf bifurcations of the general quadratic jerk system, and apply these theoretical results to a special jerk system in order to predict chaos. First, we characterize the zero-Hopf bifurcations of the general quadratic jerk system in $\mathbb{R}^{3}$. The coefficient conditions on the stability of the periodic solutions are obtained via the averaging theory of first order. Next, we apply the theoretical results to a two-parameter jerk system. Finally, special attention is paid to a jerk system with one non-negative parameter $\varepsilon$ and one non-linearity. By studying the continuation of periodic solution initiating at the zero-Hopf bifurcation, we numerically find a sequence of period doubling bifurcations, which leads to the creation of chaotic attractor.
\end{abstract}

Keywords. Jerk system; Periodic solution; Zero-Hopf bifurcation; Chaos.

\section{INTRODUCTION}

It is well known that equilibria play an important role in studying the dynamics of nonlinear differential systems. For example, one method for detecting chaos is the Shilnikov's method [1], which is based on the homoclinic orbit of a saddle-focus, ensuring the existence of a horseshoe in the neighbourhood of this orbit and consequently the birth of chaotic motions. Quadratic systems are some of the simplest after linear ones. There are many examples exhibiting rich dynamical behaviors; see, e.g., the Lorenz system [2], the Chen system [3], the Liu system [4], the Rössler system[5], the Rikitake system[6], the Lü system[7], the Maxwell-Bloch system [8], etc.

${ }^{*}$ Corresponding author.

E-mail addresses: sangbo_76@163.com (B. Sang), rizgar.salih@uor.edu.krd (R. Salih), cczuwangning@163. com (N. Wang).

Received February 29, 2020; Accepted June 10, 2020.

(C)2020 Journal of Nonlinear Functional Analysis 
Let us consider the general quadratic jerk system

$$
\left\{\begin{array}{l}
\frac{d x}{d t}=y \\
\frac{d y}{d t}=z \\
\frac{d z}{d t}=a_{0}+a_{1} x+a_{2} y+a_{3} z+a_{4} x^{2}+a_{5} y x+a_{6} z x+a_{7} y^{2}+a_{8} z y+a_{9} z^{2}
\end{array}\right.
$$

where $a_{i} \in \mathbb{R}, 0 \leq i \leq 9$. It contains some particular cases, such as, the normal form of the triple-zero bifurcation [9], the Genesio system[10], the Michelson system [11, 12] and its generalization [13]. For the special case $a_{4}=a_{5}=a_{6}=0$, it was proved in [14] that the solution and its derivatives are bounded. Many cases of system (1.1) can exhibit self-excited attractors or hidden attractors under parameter variation.

Let $\Delta=a_{1}^{2}-4 a_{0} a_{4}$ and $a_{4} \neq 0$. The system has no real equilibria if $\Delta<0$; the situation with a hidden attractor [15] is a special case. It has a single non-hyperbolic equilibrium if $\Delta=0$ for some chaotic examples; see [16,17]. Furthermore, if $a_{4}=0$, the system also has a single equilibrium. For some chaotic examples with a stable equilibrium, we refer to see [18]. For the simplest chaotic case with an unstable equilibrium, we refer to [19].

In this paper, we focus our attention on the case: $a_{4} \neq 0$. Without loss of generality, from now on, we assume $a_{4}>0$ in system (1.1). In general, a zero-Hopf equilibrium is a singular point of the 3D system, which has a zero eigenvalue and a pair of purely imaginary eigenvalues; the zero-Hopf bifurcation is a two-parameter unfolding of the system with a zero-Hopf equilibrium if the two parameters cross through the critical values. Some complicated invariant sets of the unfolding can be bifurcated from the isolated zero-Hopf equilibrium under some conditions, which may implies a local birth of chaos; see [20,21] and the reference therein.

The study of zero-Hopf bifurcations in low dimensional systems has attracted intense interest in recent years; see, e.g., $[22,23]$. One approach is to pass to normal form, but it requires some efforts; see [24] for more details. Here, we will study the zero-Hopf bifurcations of the jerk system via the averaging theory of the first order. For the averaging theory of first order and second order, we refer to $[25,26]$. For the averaging theory of other orders, we refer to $[27,28,29]$. As far as we know, the study of zero-Hopf bifurcations of system (1.1) has not been completely made in the current literature, that is, only some special cases are considered; see, e.g., [13, 16, 23]. In [16], Wei, Zhang and Yao proved the existence of zero-Hopf bifurcation at the origin of the following system

$$
\left\{\begin{array}{l}
\frac{d x}{d t}=y \\
\frac{d y}{d t}=z \\
\frac{d z}{d t}=a_{2} y+a_{3} z+a_{4} x^{2}+a_{5} x y+a_{6} x z+a_{7} y^{2}
\end{array}\right.
$$


where $a_{3}^{2}+4 a_{2}<0, a_{4} \neq 0$, for which the resulting periodic solution is unstable. In [23], Llibre and Makhlouf studied the zero-Hopf bifurcation at the origin of the following system

$$
\left\{\begin{array}{l}
\frac{d x}{d t}=y \\
\frac{d y}{d t}=z \\
\frac{d z}{d t}=\left(a_{3}+a_{6} x\right) z+\left(a_{2}+a_{5} x\right) y-x^{2}
\end{array}\right.
$$

provided an estimate of the periodic solutions and their kind of stability.

We now recall the classical averaging theory. Consider the following initial value problems

$$
\frac{d x}{d t}=\varepsilon F_{1}(t, x)+\varepsilon^{2} F_{2}(t, x, \varepsilon), \quad x(0)=x_{0},
$$

and

$$
\frac{d y}{d t}=\varepsilon g(y), \quad y(0)=x_{0},
$$

with $x, y \in D \subseteq \mathbf{R}^{n}, t \geq 0$ and $\varepsilon \in\left(0, \varepsilon_{0}\right]$. Moreover, we assume that $F_{1}, F_{2}$ are $T$-periodic in $t$, and

$$
g(y)=\frac{1}{T} \int_{0}^{T} F_{1}(t, y) d t .
$$

Theorem 1.1. [26] Consider systems (1.4) and (1.5), and suppose that:

(1) $F_{1}, D_{x} F_{1}, D_{x x} F_{1}$ and $D_{x} F_{2}$ are continuous and bounded by a constant (independent of $\varepsilon$ ) in $[0, \infty) \times D$.

(2) $F_{1}$ and $F_{2}$ are $T$-periodic in $t$, with $T$ independent of $\varepsilon$.

(3) $y(t)$ is contained in an ( $\varepsilon$-independent) interior subset of $D$ on the time-scale $1 / \varepsilon$.

Then we have the following statements:

(a) $x(t)-y(t)=\mathscr{O}(\varepsilon)$ as $\varepsilon \rightarrow 0$ on the time-scale $1 / \varepsilon$.

(b) If $p$ is an equilibrium of the averaged system (1.5) whereas

$$
\operatorname{Det} D_{y} g(p) \neq 0 \text {, }
$$

then there exists a T-periodic solution $\varphi(t, \varepsilon)$ of system (1.4), which is close to p such that

$$
\lim _{\varepsilon \rightarrow 0} \varphi(t, \varepsilon)=p
$$

(c) The stability or instability of the periodic solution $\varphi(t, \varepsilon)$ is the same as that of the equilibrium $p$. In fact, the equilibrium $p$ has the stability behaviour of the Poincaré map associated to the periodic solution $\varphi(t, \varepsilon)$.

The paper is organized as follows. In the next section, Section 2, the zero-Hopf bifurcation of a 3D system is studied. In Section 3, the results derived in the previous section are applied to system (1.1). Finally, as an application, we consider the zero-Hopf bifurcations and chaos of some special systems. 


\section{THE ZERO-HOPF BIFURCATION OF A 3D SYSTEM}

Consider the following system

$$
\left\{\begin{array}{l}
\frac{d u}{d t}=-\delta v \\
\frac{d v}{d t}=\delta u+\alpha_{1} u^{2}+\alpha_{2} v u+\alpha_{3} w u+\alpha_{4} \varepsilon u+\alpha_{5} v^{2}+\alpha_{6} w v+\alpha_{7} \varepsilon v+\alpha_{8} w^{2}+\alpha_{9} \varepsilon w, \\
\frac{d w}{d t}=\beta\left(\alpha_{1} u^{2}+\alpha_{2} v u+\alpha_{3} w u+\alpha_{4} \varepsilon u+\alpha_{5} v^{2}+\alpha_{6} w v+\alpha_{7} \varepsilon v+\alpha_{8} w^{2}+\alpha_{9} \varepsilon w\right),
\end{array}\right.
$$

where $\delta, \beta>0, \alpha_{i} \in \mathbb{R}, 1 \leq i \leq 9, \alpha_{6}, \alpha_{9} \neq 0, \alpha_{8}>0$, and $\varepsilon \in\left[0, \varepsilon_{0}\right)$ with $\varepsilon_{0}$ small enough. For this system, we consider the linear stability and the zero-Hopf bifurcation of the equilibrium $O(0,0,0)$.

Proposition 2.1. If $\alpha_{7}<0, \alpha_{9}<0$, and $\varepsilon>0$ is sufficiently small, then the origin of system (2.1) is asymptotically stable.

Proof. The characteristic polynomial of system (2.1) at the origin is

$$
P(\lambda)=\lambda^{3}-\varepsilon\left(\beta \alpha_{9}+\alpha_{7}\right) \lambda^{2}+\left(\alpha_{4} \varepsilon+\delta\right) \delta \lambda-\varepsilon \alpha_{9} \beta \delta^{2} .
$$

According to the Routh-Hurwitz criterion and the assumptions $\varepsilon, \delta, \beta>0$ for system (2.1), the origin is asymptotically stable if

$$
\alpha_{7}<-\frac{\alpha_{4} \alpha_{9} \beta \varepsilon}{\alpha_{4} \varepsilon+\delta}, \quad \alpha_{9}<0, \quad \alpha_{4}>-\frac{\delta}{\varepsilon} .
$$

Since $\varepsilon>0$ is sufficiently small, the origin is asymptotically stable.

System (2.1) has an isolated zero-Hopf equilibrium at the origin for $\varepsilon=0$. In the next section, this system will be shown to be a prototype for studying the zero-Hopf bifurcations of system (1.1).

Under the cylindrical coordinates,

$$
u=r \cos (\theta), v=r \sin (\theta), w=w,
$$

where $r>0$, system (2.1) becomes

$$
\left\{\begin{array}{l}
\frac{d r}{d t}=\sin \theta\left(p_{1}(\theta) r^{2}+p_{2}(\theta) w r+p_{3}(\theta) \varepsilon r+\alpha_{8} w^{2}+\alpha_{9} \varepsilon w\right) \\
\frac{d \theta}{d t}=\delta+q_{1}(\theta) r+q_{2}(\theta) \frac{w^{2}}{r}+q_{3}(\theta) \frac{\varepsilon w}{r}+q_{4}(\theta) w+q_{5}(\theta) \varepsilon \\
\frac{d w}{d t}=\beta\left(p_{1}(\theta) r^{2}+p_{2}(\theta) w r+p_{3}(\theta) \varepsilon r+\alpha_{8} w^{2}+\alpha_{9} \varepsilon w\right)
\end{array}\right.
$$

where

$$
\begin{aligned}
& p_{1}(\theta)=\left(\alpha_{5}-\alpha_{1}\right) \sin ^{2} \theta+\alpha_{2} \sin \theta \cos \theta+\alpha_{1}, \\
& p_{2}(\theta)=\alpha_{3} \cos \theta+\alpha_{6} \sin \theta, \\
& p_{3}(\theta)=\alpha_{4} \cos \theta+\alpha_{7} \sin \theta,
\end{aligned}
$$


and

$$
\begin{aligned}
& q_{1}(\theta)=-\alpha_{2} \sin ^{3} \theta+\left(\alpha_{5}-\alpha_{1}\right) \cos \theta \sin ^{2} \theta+\alpha_{2} \sin \theta+\alpha_{1} \cos \theta \\
& q_{2}(\theta)=\alpha_{8} \cos \theta \\
& q_{3}(\theta)=\alpha_{9} \cos \theta \\
& q_{4}(\theta)=-\alpha_{3} \sin ^{2} \theta+\alpha_{6} \cos \theta \sin \theta+\alpha_{3} \\
& q_{5}(\theta)=-\alpha_{4} \sin ^{2} \theta+\alpha_{7} \cos \theta \sin \theta+\alpha_{4}
\end{aligned}
$$

Using the rescaling $r=\varepsilon R, w=\varepsilon W$, system (2.5) becomes

$$
\left\{\begin{array}{l}
\frac{d R}{d t}=\varepsilon \sin \theta\left(p_{1}(\theta) R^{2}+p_{2}(\theta) W R+p_{3}(\theta) R+\alpha_{8} W^{2}+\alpha_{9} W\right) \\
\frac{d \theta}{d t}=\delta+\varepsilon\left(q_{1}(\theta) R+q_{2}(\theta) \frac{W^{2}}{R}+q_{3}(\theta) \frac{W}{R}+q_{4}(\theta) W+q_{5}(\theta)\right) \\
\frac{d W}{d t}=\varepsilon \beta\left(p_{1}(\theta) R^{2}+p_{2}(\theta) W R+p_{3}(\theta) R+\alpha_{8} W^{2}+\alpha_{9} W\right) .
\end{array}\right.
$$

Therefore, the solutions of system (2.14) in the region $\frac{d \theta}{d t}>0$ can be studied through the solutions of the following system

$$
\left\{\begin{array}{l}
\frac{d R}{d \theta}=F_{1}(\theta, R, W) \varepsilon+O\left(\varepsilon^{2}\right) \\
\frac{d W}{d \theta}=F_{2}(\theta, R, W) \varepsilon+O\left(\varepsilon^{2}\right)
\end{array}\right.
$$

where

$$
\begin{aligned}
& F_{1}(\theta, R, W)=\frac{\sin \theta\left(p_{1}(\theta) R^{2}+p_{2}(\theta) W R+p_{3}(\theta) R+\alpha_{8} W^{2}+\alpha_{9} W\right)}{\delta}, \\
& F_{2}(\theta, R, W)=\frac{\beta\left(p_{1}(\theta) R^{2}+p_{2}(\theta) W R+p_{3}(\theta) R+\alpha_{8} W^{2}+\alpha_{9} W\right)}{\delta} .
\end{aligned}
$$

Using the same notations as in Theorem 1.1, we have $t=\theta, T=2 \pi, x=(R, W)^{T}$, and

$$
F(\theta, R, W)=\left(\begin{array}{c}
F_{1}(\theta, R, W) \\
F_{2}(\theta, R, W)
\end{array}\right), \quad f(R, W)=\left(\begin{array}{c}
f_{1}(R, W) \\
f_{2}(R, W)
\end{array}\right),
$$

where

$$
f_{i}(R, W)=\frac{1}{2 \pi} \int_{0}^{2 \pi} F_{i}(\theta, R, W) d \theta, \quad i=1,2 .
$$

Thus, for system (2.15), we have

$$
\begin{aligned}
f_{1}(R, W) & =\frac{R\left(\alpha_{6} W+\alpha_{7}\right)}{2 \delta}, \\
f_{2}(R, W) & =\frac{\beta\left[\left(\alpha_{1}+\alpha_{5}\right) R^{2}+2 \alpha_{8} W^{2}+2 \alpha_{9} W\right]}{2 \delta} .
\end{aligned}
$$

Hence the averaged system reads

$$
\left\{\begin{array}{l}
\frac{d R}{d \theta}=f_{1}(R, W) \varepsilon \\
\frac{d W}{d \theta}=f_{2}(R, W) \varepsilon
\end{array}\right.
$$


and therefore the unique equilibrium of (2.22) with $R>0$ is

$$
\left(R^{*}, W^{*}\right)=\left(\frac{1}{\left|\alpha_{6}\right|} \sqrt{\frac{2 \alpha_{7}\left(\alpha_{6} \alpha_{9}-\alpha_{7} \alpha_{8}\right)}{\alpha_{1}+\alpha_{5}}},-\frac{\alpha_{7}}{\alpha_{6}}\right),
$$

where

$$
\alpha_{7}\left(\alpha_{6} \alpha_{9}-\alpha_{7} \alpha_{8}\right)\left(\alpha_{1}+\alpha_{5}\right)>0 .
$$

Computing the Jacobian of $f_{1}, f_{2}$ at this point, we get

$$
\left.\operatorname{det} \frac{\partial\left(f_{1}, f_{2}\right)}{\partial(R, W)}\right|_{(R, W)=\left(R^{*}, W^{*}\right)}=-\frac{\alpha_{7}\left(\alpha_{6} \alpha_{9}-\alpha_{7} \alpha_{8}\right) \beta}{\alpha_{6} \delta^{2}},
$$

which is non-zero due to $\beta>0$ and (2.24). We consider the stability of the equilibrium $\left(R^{*}, W^{*}\right)$, for which the characteristic polynomial is

$$
P(\lambda)=\lambda^{2}+b_{1} \lambda+b_{2}
$$

where

$$
\begin{aligned}
& b_{1}=-\frac{\beta\left(\alpha_{6} \alpha_{9}-2 \alpha_{7} \alpha_{8}\right)}{\alpha_{6} \delta}, \\
& b_{2}=-\frac{\beta\left(\alpha_{6} \alpha_{9}-\alpha_{7} \alpha_{8}\right) \alpha_{7}}{\delta^{2} \alpha_{6}} .
\end{aligned}
$$

It is obvious that $b_{2} \neq 0$ due to $\beta>0$ and (2.24). Thus $\left(R^{*}, W^{*}\right)$ is non-degenerate. According to the Routh-Hurwitz criterion, the polynomial (2.26) has all its roots with negative real parts if and only if

$$
b_{1}>0, \quad b_{2}>0,
$$

which implies that equilibrium $\left(R^{*}, W^{*}\right)$ is an attractor. Using the Trace-Determinant criteria, the equilibrium is a repellor if

$$
b_{1}<0, \quad b_{2}>0
$$

and it is a saddle point if

$$
b_{2}<0 \text {. }
$$

Consider the case $\alpha_{9}>0$. Note condition (2.24) and the basic assumptions $\beta>0, \delta>0, \alpha_{8}>$ 0 for system (2.1). By solving (2.27), (2.28) and (2.29), respectively, we get the following conclusions:

(a) The equilibrium is an attractor if one of the following conditions hold:

$$
\begin{array}{lll}
\text { (a }) \alpha_{6}<0, & \alpha_{7}<0, & \frac{\alpha_{9} \alpha_{6}}{2 \alpha_{7}}<\alpha_{8}<\frac{\alpha_{9} \alpha_{6}}{\alpha_{7}} \\
\text { (a) } \alpha_{6}>0, & \alpha_{7}>0, & \alpha_{8}>\frac{\alpha_{9} \alpha_{6}}{\alpha_{7}}
\end{array}
$$

(b) The equilibrium is a repellor if one of the following conditions hold:
(b $\left.\alpha_{1}\right) \alpha_{6}<0, \quad \alpha_{7}<0, \quad \alpha_{8}<\frac{\alpha_{6} \alpha_{9}}{2 \alpha_{7}}$;
$\left(\mathrm{b}_{2}\right) \alpha_{6}>0, \quad \alpha_{7}<0$.

(c) The equilibrium is a saddle point if one of the following conditions holds:

$$
\begin{aligned}
& \text { (c } 1) \alpha_{6}, \alpha_{7}<0, \quad \alpha_{8}>\frac{\alpha_{6} \alpha_{9}}{\alpha_{7}} ; \\
& \text { (c } \left.\alpha_{2}\right) \alpha_{6}<0, \quad \alpha_{7}>0 ;
\end{aligned}
$$


(с $\left.\alpha_{3}\right) \alpha_{6}, \alpha_{7}>0, \quad \alpha_{8}<\frac{\alpha_{9} \alpha_{6}}{\alpha_{7}}$.

Theorem 2.2. Assume that $\alpha_{9}>0$ and (2.24). Then, for system (2.1), a periodic solution borns at the origin when $\varepsilon=0$, and it exists for $\varepsilon>0$ sufficiently small. Moreover, the following statements hold:

(1) In case (a), the periodic solution is asymptotically stable;

(2) In case (b), the periodic solution is unstable;

(3) In case (c), the periodic solution has a stable and an unstable manifolds, which locally are formed by two cylinders.

Proof. According to Theorem 1.1, for $\varepsilon>0$ and sufficiently small, system (2.15) has a periodic solution $(R(\theta, \varepsilon), W(\theta, \varepsilon))$ such that

$$
(R(0, \varepsilon), W(0, \varepsilon))=\left(R^{*}, W^{*}\right)+\mathscr{O}(\varepsilon) .
$$

Using the coordinates $(R, \theta, W)$, the periodic solution $(R(t, \varepsilon), \theta(t, \varepsilon), W(t, \varepsilon))$ of system (2.14) satisfies

$$
(R(0, \varepsilon), \theta(0, \varepsilon), W(0, \varepsilon))=\left(R^{*}, 0, W^{*}\right)+\mathscr{O}(\varepsilon) .
$$

Now the periodic solution $(r(t, \varepsilon), \theta(t, \varepsilon), w(t, \varepsilon))$ of system (2.5) satisfies

$$
(r(0, \varepsilon), \theta(0, \varepsilon), w(0, \varepsilon))=\left(\varepsilon R^{*}, 0, \varepsilon W^{*}\right)+\mathscr{O}\left(\varepsilon^{2}\right) .
$$

Finally, in system (2.1), the periodic solution $(u(t, \varepsilon), v(t, \varepsilon), w(t, \varepsilon))$ satisfies

$$
(u(0, \varepsilon), v(0, \varepsilon), w(0, \varepsilon))=\left(\varepsilon R^{*}, 0, \varepsilon W^{*}\right)+\mathscr{O}\left(\varepsilon^{2}\right) .
$$

The stability of this solution follows from that of the equilibrium $\left(R^{*}, W^{*}\right)$ in the averaged system (2.22). Thus we complete the proof.

Similarly, the conclusions for the case $\alpha_{9}<0$ are listed below:

(d) The equilibrium is an attractor if one of the following conditions holds:
(d $\left.\mathrm{d}_{1}\right) \alpha_{6}<0$
$\alpha_{7}>0, \quad \alpha_{8}<\frac{\alpha_{9} \alpha_{6}}{2 \alpha_{7}}$
(d $\left.\mathrm{d}_{2}\right) \alpha_{6}>0$
$\alpha_{7}>0$.

(e) The equilibrium is a repellor if one of the following conditions holds:
(e $\left.e_{1}\right)<0$
$\alpha_{7}>0$
$\frac{\alpha_{9} \alpha_{6}}{2 \alpha_{7}}<\alpha_{8}<\frac{\alpha_{9} \alpha_{6}}{\alpha_{7}}$
$\left(\mathrm{e}_{2}\right) \alpha_{6}>0$
$\alpha_{7}<0$
$\alpha_{8}>\frac{\alpha_{9} \alpha_{6}}{\alpha_{7}}$

(f) The equilibrium is a saddle point if one of the following conditions holds:

(f $\left.\mathrm{f}_{1}\right) \alpha_{6}<0, \quad \alpha_{7}<0$;

(f) $\alpha_{6}>0, \quad \alpha_{7}<0, \quad \alpha_{8}<\frac{\alpha_{9} \alpha_{6}}{\alpha_{7}}$;

(f $\left.\mathrm{f}_{3}\right) \alpha_{6}<0, \quad \alpha_{7}>0, \quad \alpha_{8}>\frac{\alpha_{9} \alpha_{6}}{\alpha_{7}}$.

Using the same arguments, we can concluded that the following.

Theorem 2.3. Assume that $\alpha_{9}<0$ and (2.24). Then for system (2.1), a periodic solution borns at the origin when $\varepsilon=0$, and it exists for $\varepsilon>0$ sufficiently small. Moreover, the following statements hold:

(1) In case (d), the periodic solution is asymptotically stable. 
(2) In case (e), the periodic solution is unstable.

(3) In case (f), the periodic solution has a stable and an unstable manifolds, which locally are formed by two cylinders.

Remark 2.4. According to Proposition 2.1, there are three sub-cases for system (2.1): $\left(\mathrm{e}_{2}\right),\left(\mathrm{f}_{1}\right)$ and $\left(\mathrm{f}_{2}\right)$, in which the origin is asymptotically stable when $\varepsilon>0$ is sufficiently small.

Remark 2.5. Recall the expressions for $f_{1}(R, W), f_{2}(R, W)$ in (2.20) and (2.21), and the assumptions for (2.1) at the beginning of this section: $\alpha_{6}, \alpha_{9} \neq 0$ and $\alpha_{8}>0$. So $f_{1}(R, W)$, $f_{2}(R, W)$ cannot be identically zero. Thus there is no need for considering the averaging theory of second order.

\section{ZERO-HOPF BIFURCATIONS IN SYSTEM (1.1)}

Recalling that we have assumed that $a_{4}>0$ in the introduction section. Thus system (1.1) has two equilibria, $E_{1}=\left(x_{1}, 0,0\right)$ and $E_{2}=\left(x_{2}, 0,0\right)$, where

$$
x_{1}=-\frac{a_{1}}{2 a_{4}}+\frac{\sqrt{\Delta}}{2 a_{4}}, \quad x_{2}=-\frac{a_{1}}{2 a_{4}}-\frac{\sqrt{\Delta}}{2 a_{4}},
$$

where $\Delta=a_{1}^{2}-4 a_{0} a_{4}$.

Proposition 3.1. There is a eight-parameter family of system (1.1), for which the equilibria $E_{1,2}$ are both zero-Hopf equilibria, that is,

$$
a_{0}=\frac{a_{1}^{2}}{4 a_{4}}, \quad a_{3}=\frac{a_{6} a_{1}}{2 a_{4}}
$$

with

$$
a_{2}<\frac{a_{1} a_{5}}{2 a_{4}} .
$$

The two equilibria merge into a single equilibrium $\left(-\frac{a_{1}}{2 a_{4}}, 0,0\right)$ at the bifurcation, with the spectrum $\lambda_{1,2}= \pm \mathrm{i} \sqrt{\frac{a_{1} a_{5}}{2 a_{4}}-a_{2}}, \quad \lambda_{3}=0$.

Proof. The Jacobian matrix at $E_{1}$ is

$$
A=\left[\begin{array}{ccc}
0 & 1 & 0 \\
0 & 0 & 1 \\
2 a_{4} x_{1}+a_{1} & a_{5} x_{1}+a_{2} & a_{6} x_{1}+a_{3}
\end{array}\right],
$$

whose characteristic polynomial is

$$
P(\lambda)=\lambda^{3}+g_{2} \lambda^{2}+g_{1} \lambda+g_{0}
$$

where

$$
g_{0}=-2 a_{4} x_{1}-a_{1}, \quad g_{1}=-a_{5} x_{1}-a_{2}, \quad g_{2}=-a_{6} x_{1}-a_{3} .
$$

By imposing the conditions for the zero-Hopf bifurcation, i.e., $g_{0}=g_{2}=0$ and $g_{1}>0$. After some tedious algebra, we obtain the conditions (3.2) and (3.3). The analysis for $E_{2}$ is similar, and we omit it here. The rest part of this proposition can be verified easily. This completes the proof. 
Set the parameters in system (1.1) as follows:

$$
\left\{\begin{array}{l}
a_{0}=s_{0} \varepsilon^{2}+\frac{a_{1,0}{ }^{2}}{4 a_{4,0}} \\
a_{1}=s_{1} \varepsilon^{2}+a_{1,0} \\
a_{2}=s_{2} \varepsilon+a_{2,0} \\
a_{3}=s_{3} \varepsilon+\frac{a_{1,0} a_{6,0}}{2 a_{4,0}} \\
a_{4}=s_{4} \varepsilon^{2}+a_{4,0}, \\
a_{k}=s_{k} \varepsilon+a_{k, 0}, \quad 5 \leq k \leq 9
\end{array}\right.
$$

and

$$
\left\{\begin{aligned}
s_{0} & <-\frac{a_{1,0}\left(a_{1,0} s_{4}-2 a_{4,0} s_{1}\right)}{4 a_{4,0}{ }^{2}} \\
a_{4,0} & >0 \\
a_{2,0} & <\frac{a_{5,0} a_{1,0}}{2 a_{4,0}} .
\end{aligned}\right.
$$

where $\varepsilon>0$ sufficiently small, $a_{i, 0}, s_{i}, 0 \leq i \leq 9$ are all real parameters. With the foregoing conditions, $x_{1}$ and $x_{2}$ become

$$
x_{1}=-\frac{a_{1,0}}{2 a_{4,0}}+\frac{\eta \varepsilon}{2 a_{4,0}}+\mathscr{O}\left(\varepsilon^{2}\right), \quad x_{2}=-\frac{a_{1,0}}{2 a_{4,0}}-\frac{\eta \varepsilon}{2 a_{4,0}}+\mathscr{O}\left(\varepsilon^{2}\right)
$$

where

$$
\eta=\sqrt{2 a_{1,0} s_{1}-4 a_{4,0} s_{0}-\frac{a_{1,0}^{2} s_{4}}{a_{4,0}}}
$$

It should be noted that the developing of $x_{1,2}$ up to $\varepsilon^{1}$ is sufficient for applying the averaging theory of first order. Let

$$
\xi=\sqrt{2 a_{1,0} a_{4,0} a_{5,0}-4 a_{2,0} a_{4,0}{ }^{2}}
$$

Both $\xi$ and $\eta$ are positive due to the conditions (3.5).

3.1. The periodic solution arising from $E_{1}$. Under conditions (3.4) and (3.5), we can use the transformation

$$
\left\{\begin{array}{l}
x=-\frac{4 a_{4,0}^{2}}{\xi^{2}} v+w+x_{1} \\
y=-\frac{2 a_{4,0}}{\xi} u \\
z=v
\end{array}\right.
$$


which transforms system (1.1) to

$$
\left\{\begin{aligned}
\frac{d u}{d t}= & -\delta v \\
\frac{d v}{d t}= & \delta u+\alpha_{1} u^{2}+\alpha_{2} v u+\alpha_{3} w u+\alpha_{4}(\eta) \varepsilon u+\alpha_{5} v^{2}+\alpha_{6} w v \\
& +\alpha_{7}(\eta) \varepsilon v+\alpha_{8} w^{2}+\alpha_{9}(\eta) \varepsilon w+\cdots \\
\frac{d w}{d t}= & \beta\left[\alpha_{1} u^{2}+\alpha_{2} v u+\alpha_{3} w u+\alpha_{4}(\eta) \varepsilon u+\alpha_{5} v^{2}+\alpha_{6} w v\right. \\
& \left.+\alpha_{7}(\eta) \varepsilon v+\alpha_{8} w^{2}+\alpha_{9}(\eta) \varepsilon w\right]+\cdots
\end{aligned}\right.
$$

where

$$
\begin{aligned}
\delta & =\sqrt{\frac{a_{5,0} a_{1,0}}{2 a_{4,0}}-a_{2,0}}, \quad \beta=\frac{4 a_{4,0}{ }^{2}}{\xi^{2}}, \\
\alpha_{1} & =\frac{4 a_{7,0} a_{4,0} \xi^{2}}{\xi^{2}}, \\
\alpha_{2} & =-\frac{2 a_{4,0}\left(\xi^{2} a_{8,0}-4 a_{4,0} a_{5,0}\right)}{\xi^{3}}, \\
\alpha_{3} & =-\frac{2 a_{5,0} a_{4,0}}{\xi}, \\
\alpha_{4}(\eta) & =-\frac{a_{5,0} \eta-s_{5} a_{1,0}+2 s_{2} a_{4,0}}{\xi}, \\
\alpha_{5} & =\frac{a_{9,0} \xi^{4}-4 a_{6,0} a_{4,0} \xi^{2}+16 a_{4,0}}{\xi^{4}}, \\
\alpha_{6} & =\frac{a_{6,0} \xi^{2}-8 a_{4,0}{ }^{3}}{\xi^{2}}, \\
\alpha_{7}(\eta) & =\frac{a_{6,0} \eta \xi^{2}-8 \eta a_{4,0}-s_{6} a_{1,0} \xi^{2}+2 s_{3} \xi^{2} a_{4,0}}{2 \xi^{2} a_{4,0}}, \\
\alpha_{8} & =a_{4,0}, \\
\alpha_{9}(\eta) & =\eta,
\end{aligned}
$$

and we have dropped terms that are not used in the following analysis.

Now the system (3.10) is in the form of (2.1) with $\alpha_{9}(\eta)=\eta>0$. Applying Theorem 2.2 to it, we get the following conclusion.

Theorem 3.2. Let $\alpha_{i}=\alpha_{i}(\eta), i=4,7,9$ and assume that the conditions (2.24), (3.4), and (3.5) hold. Then for system (1.1), a periodic solution borns at $E_{1}$ when $\varepsilon=0$, and it exists for $\varepsilon>0$ sufficiently small. Moreover, the following statements hold:

in case (a), the periodic solution is asymptotically stable;

in case (b), the periodic solution is unstable;

in case (c), the periodic solution has a stable and an unstable manifolds, which locally are formed by two cylinders. 
3.2. The periodic solution arising from $E_{2}$. Under conditions (3.4) and (3.5), we can use the transformation

$$
\left\{\begin{array}{l}
x=-\frac{4 a_{4,0}^{2}}{\xi^{2}} v+w+x_{2}, \\
y=-\frac{2 a_{4,0}}{\xi} u \\
z=v
\end{array}\right.
$$

which transforms system (1.1) to

$$
\left\{\begin{aligned}
\frac{d u}{d t}= & -\delta v \\
\frac{d v}{d t}= & \delta u+\alpha_{1} u^{2}+\alpha_{2} v u+\alpha_{3} w u+\alpha_{4}(-\eta) \varepsilon u+\alpha_{5} v^{2}+\alpha_{6} w v \\
& +\alpha_{7}(-\eta) \varepsilon v+\alpha_{8} w^{2}+\alpha_{9}(-\eta) \varepsilon w+\cdots \\
\frac{d w}{d t}= & \beta\left[\alpha_{1} u^{2}+\alpha_{2} v u+\alpha_{3} w u+\alpha_{4}(-\eta) \varepsilon u+\alpha_{5} v^{2}+\alpha_{6} w v\right. \\
& \left.+\alpha_{7}(-\eta) \varepsilon v+\alpha_{8} w^{2}+\alpha_{9}(-\eta) \varepsilon w\right]+\cdots
\end{aligned}\right.
$$

System (3.22) is in the form of (2.1) with $\alpha_{9}(-\eta)=-\eta<0$. Applying Theorem 2.3 to it, we get the following conclusions.

Theorem 3.3. Let $\alpha_{i}=\alpha_{i}(-\eta), i=4,7,9$ and assume that the conditions (2.24), (3.4), and (3.5) hold. Then, for system (1.1), a periodic solution borns at $E_{2}$ when $\varepsilon=0$, and it exists for $\varepsilon>0$ sufficiently small. Moreover, the following statements hold:

in case $(d)$, the periodic solution is asymptotically stable;

in case (e), the periodic solution is unstable;

in case $(f)$, the periodic solution has a stable and an unstable manifolds, which locally are formed by two cylinders.

\section{ZERO-HOPF BIFURCATION AND CHAOS FOR A CLASS OF SYSTEMS}

From (3.2), we know that $a_{0}$ and $a_{3}$ are the parameters of the zero-Hopf bifurcations in system (1.1). Therefore, we may choose $a_{1}=a_{5}=a_{6}=a_{7}=a_{8}=a_{9}=0, a_{2}=-7$ and $a_{4}=1$. This yields the following system

$$
\left\{\begin{array}{l}
\frac{d x}{d t}=y, \\
\frac{d y}{d t}=z, \\
\frac{d z}{d t}=a_{0}-7 y+a_{3} z+x^{2},
\end{array}\right.
$$

where we may assume $a_{0} \leq 0$. It belongs to $J D_{2}$; see [30, 31].

According to Proposition 3.1, zero-Hopf bifurcations occur simultaneously at

$$
a_{0}=0, \quad a_{3}=0
$$


for the equilibria $E_{1}=\left(\sqrt{-a_{0}}, 0,0\right), E_{2}=\left(-\sqrt{-a_{0}}, 0,0\right)$ of system (4.1). At this bifurcation point, system (4.1) is reduced to

$$
\left\{\begin{array}{l}
\frac{d x}{d t}=y, \\
\frac{d y}{d t}=z \\
\frac{d z}{d t}=-7 y+x^{2}
\end{array}\right.
$$

and the two equilibria merge into a single equilibrium $O(0,0,0)$. As in Section 3 , we consider the following perturbed system

$$
\left\{\begin{array}{l}
\frac{d x}{d t}=y \\
\frac{d y}{d t}=z \\
\frac{d z}{d t}=a_{0}+a_{2} y+a_{3} z+a_{4} x^{2}
\end{array}\right.
$$

where

$$
\left\{\begin{array}{l}
a_{0}=s_{0} \varepsilon^{2}, \\
a_{2}=s_{2} \varepsilon-7, \\
a_{3}=s_{3} \varepsilon, \\
a_{4}=s_{4} \varepsilon^{2}+1, \\
s_{0}<0 .
\end{array}\right.
$$

This is a special case of system (1.1), and the other parameters $a_{1}, a_{5}, a_{6}, a_{7}, a_{8}, a_{9}$ in the form of (3.4) are identically zero. As in Subsection 3.1, the involved quantities are

$$
\begin{aligned}
\eta & =2 \sqrt{-s_{0}}, \quad \xi=2 \sqrt{7}, \quad \delta=\sqrt{7}, \quad \beta=\frac{4}{\xi^{2}}, \\
\alpha_{1} & =\alpha_{2}=\alpha_{3}=0, \quad \alpha_{4}(\eta)=\alpha_{4}(-\eta)=-\frac{2 s_{2}}{\xi}, \quad \alpha_{5}=\frac{16}{\xi^{4}}, \quad \alpha_{6}=-\frac{8}{\xi^{2}}, \\
\alpha_{7}(\eta) & =-\frac{4 \eta}{\xi^{2}}+s_{3}, \quad \alpha_{7}(-\eta)=\frac{4 \eta}{\xi^{2}}+s_{3}, \quad \alpha_{8}=1, \quad \alpha_{9}(\eta)=\eta, \quad \alpha_{9}(-\eta)=-\eta .
\end{aligned}
$$

With the aid of conditions (4.5), the equilibria of system (4.4) become $E_{1}=\left(x_{1}, 0,0\right)$ and $E_{2}=\left(x_{2}, 0,0\right)$, where

$$
x_{1}=\frac{\eta \varepsilon}{2}+\mathscr{O}\left(\varepsilon^{2}\right), \quad x_{2}=-\frac{\eta \varepsilon}{2}+\mathscr{O}\left(\varepsilon^{2}\right),
$$

are changing with respect to $\varepsilon$. Applying t Theorems 3.2 and 3.3 to system (4.4), we get the following conclusions, respectively.

Theorem 4.1. Let $\alpha_{i}=\alpha_{i}(\eta), i=4,7,9$ and assume that the conditions (2.24) and (4.5) hold. Then, for system (4.4), a periodic solution borns at $E_{1}$ when $\varepsilon=0$, and it exists for $\varepsilon>0$ sufficiently small. Moreover, the following statements hold:

in case $\left(a_{1}\right)$, the periodic solution is asymptotically stable;

in case $\left(b_{1}\right)$, the periodic solution is unstable; 
in case $\left(c_{1}\right)$ or $\left(c_{2}\right)$, the periodic solution has a stable and an unstable manifolds, which locally are formed by two cylinders.

Theorem 4.2. Let $\alpha_{i}=\alpha_{i}(-\eta), i=4,7,9$ and assume that the conditions (2.24) and (4.5) hold. Then, for system (4.4), a periodic solution borns at $E_{2}$ when $\varepsilon=0$, and it exists for $\varepsilon>0$ sufficiently small. Moreover, the following statements hold:

in case $\left(d_{1}\right)$, the periodic solution is asymptotically stable;

in case $\left(e_{1}\right)$, the periodic solution is unstable;

in case $\left(f_{1}\right)$ or $\left(f_{3}\right)$, the periodic solution has a stable and an unstable manifolds, which locally are formed by two cylinders.

Consider the following system

$$
\left\{\begin{array}{l}
\frac{d x}{d t}=y \\
\frac{d y}{d t}=z \\
\frac{d z}{d t}=-\varepsilon^{2}-7 y-0.129 \varepsilon z+x^{2}
\end{array}\right.
$$

where $\varepsilon \geq 0$ is a parameter. It contains only one control parameter and only one non-linearity.

The system has two equilibria $E_{1,2}=( \pm \varepsilon, 0,0)$. For them, zero-Hopf bifurcations occur at $\varepsilon=0$. Furthermore, Hopf bifurcation can not occur. It is easy to check that the condition $\left(a_{1}\right)$ in Theorem 4.1 is satisfied. Thus, according to the theoretical expectations, there exists a stable periodic solution near the origin for $\varepsilon$ small. This is shown in Fig. 1 for $\varepsilon=0.02$.

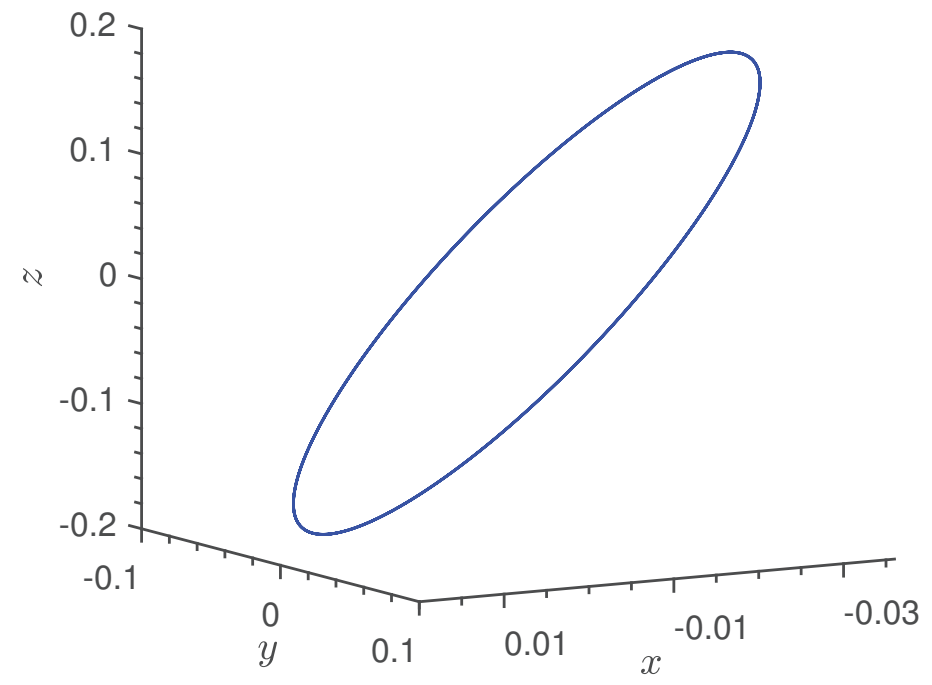

FIGURE 1. Periodic solution of system (4.7) when $\varepsilon=0.02$. Initial conditions are given by $x(0)=y(0)=z(0)=0$

When $\varepsilon=7.75$, the system becomes chaotic. The Lyapunov exponents of the system are $L_{1}=0.27047, L_{2}=0, L_{3}=-1.2703$, so that the Kaplan-Yorke dimension is $D_{\mathrm{KY}}=2.213$. It is a novel chaotic system, which is not reported in [30, 31, 32]. The largest Lyapunov exponent of it is relatively bigger than the known chaotic systems in the class $J D_{2}$. See Fig. 2 for the chaotic attractor and Fig. 3(b) for the evolution of Lyapunov exponents versus $\varepsilon$. 
In Fig. 3, the bifurcation diagram for $x$ versus $\varepsilon$ is displayed for the initial conditions $x(0)=$ $y(0)=z(0)=0$ on the interval $[0,7.8]$. Starting from a zero-Hopf equilibrium, the system undergoes a zero-Hopf bifurcation at $\varepsilon=0$, period-doubling bifurcations at $\varepsilon=5.348,6.014,6.176, \cdots$, and then becomes chaotic at about $\varepsilon=6.240$. In addition to this value, there is a wide range of $\varepsilon$ for which the eventual behaviour is chaotic. Several narrow periodic windows can observed which surrounded by regions of chaos. By the way, we find a narrow range $[6.02,6.20]$ of the parameter $\varepsilon$ in which there are coexisting attractors; see Fig. 4. In a word, despite the simplicity of system (4.7), it has rich dynamical behaviours: zero-Hopf bifurcations, periodic attractors, coexistence of attractors, and chaotic attractors depending on the parameter values. The pattern for transition to chaos is zero-Hopf bifurcations $\mapsto$ periodic attractors $\mapsto$ period doubling cascades $\mapsto$ chaos.

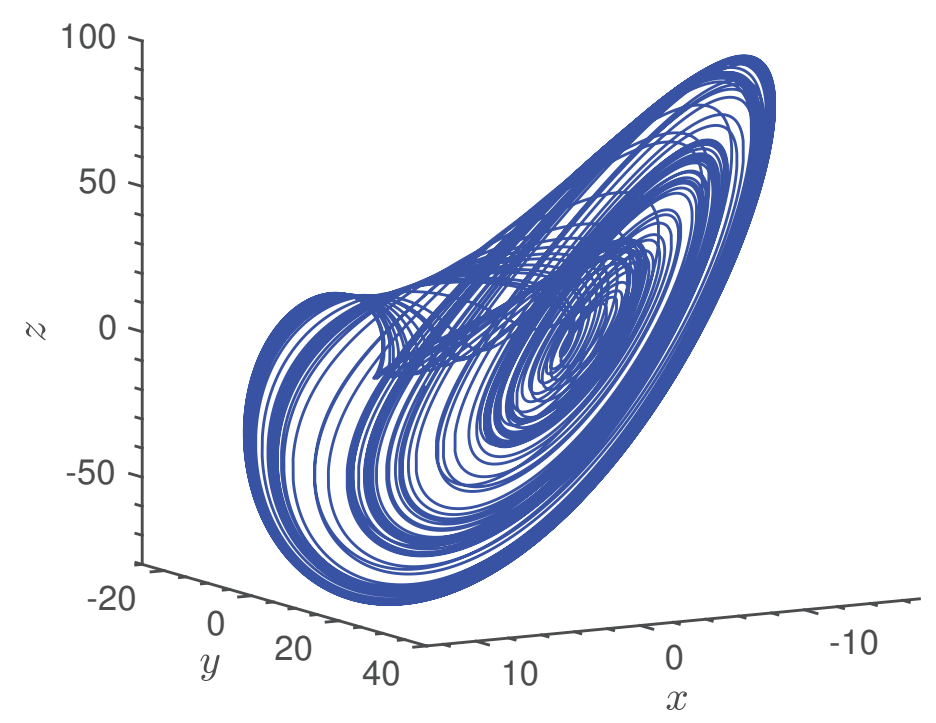

FIGURE 2. Chaotic attractor of system (4.7) when $\varepsilon=7.75$. Initial conditions are given by $x(0)=y(0)=z(0)=0$

\section{CONCLUSIONS}

In this paper, we studied the zero-Hopf bifurcations of the general quadratic jerk system. The system has ten parameters, in which one parameter is assumed to be positive. In order to apply the averaging theory of first order, we considered perturbations of the values of the parameters for which the jerk system has a zero-Hopf equilibrium. We obtained the stability conditions for the periodic solutions bifurcated from the zero-Hopf bifurcations. These conditions can help to understand the formulating mechanism of chaotic attractors in the jerk system. As an application, we studied the zero-Hopf bifurcations of some quadratic jerk systems. The obtained results were applied to a one-parameter jerk system, for which period doubling cascade, coexisting attractors and chaos with narrow periodic windows were found on varying the parameter.

\section{Funding}



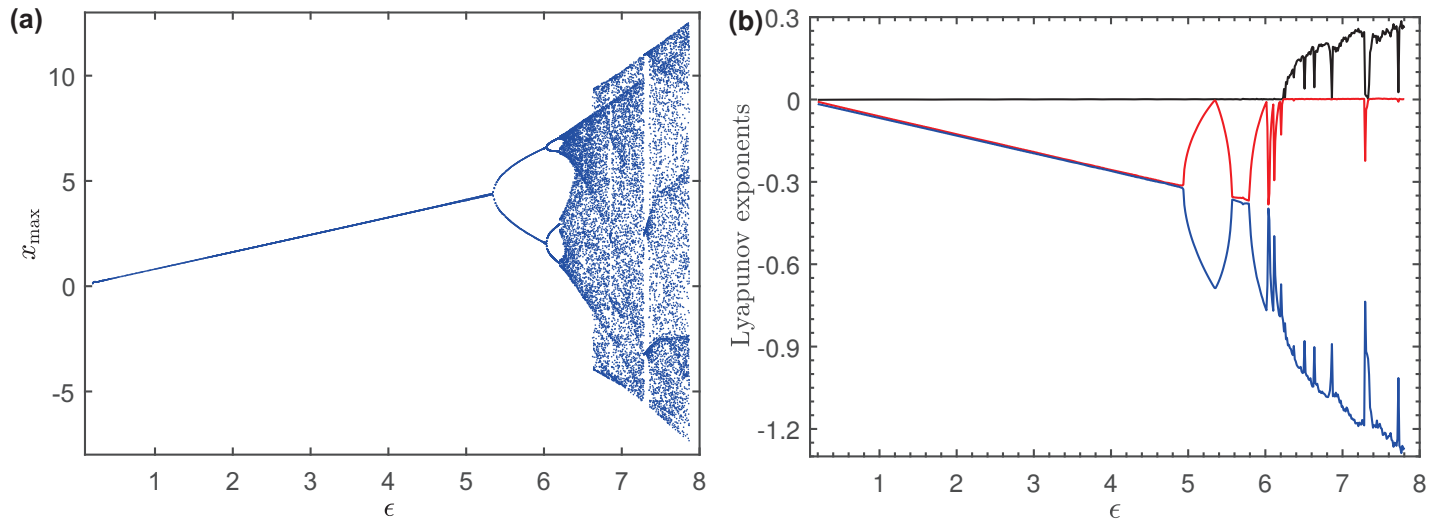

FIgURE 3. (a) Bifurcation diagram of system (4.7): maximum values of $x$ versus $\varepsilon$ on the interval [0,7.8]. The initial conditions are $x(0)=y(0)=z(0)=0$. (b) The corresponding Lyapunov exponents depicted as functions of $\varepsilon$ on the same interval
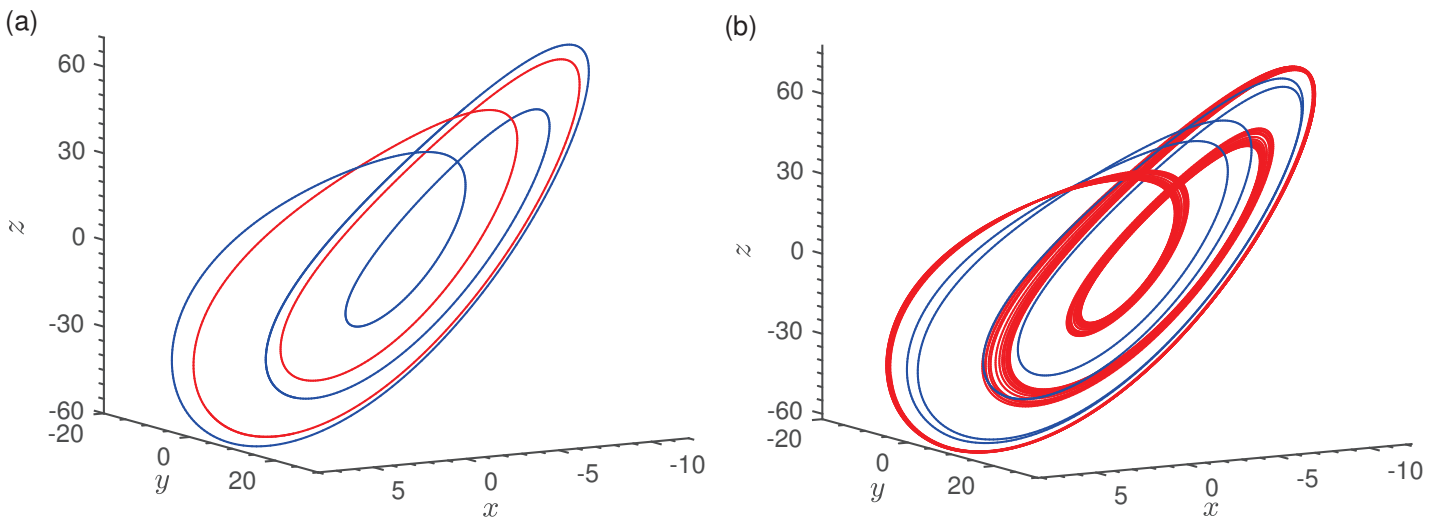

FIGURE 4. The blue attractor is generated from the initial conditions $(0,0,0)$, and the red one from the initial conditions $(-0.53,-0.64,0.22)$. (a) Coexistence of two periodic attractors with $\varepsilon=6.02$. (b) Coexistence of a periodic attractor and a chaotic attractor with $\varepsilon=6.180$. The Lyapunov exponents of the chaotic attractor are $L_{1}=0.054637, L_{2}=0, L_{3}=-0.85183$, so that the Kaplan-Yorke dimension is $D_{\mathrm{KY}}=2.0641$

The first author was supported by Shandong Provincial Natural Science Foundation, China (ZR2018MA025).

\section{REFERENCES}

[1] C.P. Silva, Shil'nikov's theorem- a tutorial, IEEE Trans. Circuits Syst.-I 40 (1993), 675-682.

[2] E.N. Lorenz, Deterministic non-periodic flow, J. Atmos. Sci. 20 (1963), 130-141.

[3] G.R. Chen, T. Ueta, Yet another chaotic attractor, Int. J. Bifur. Chaos, 9 (1999), 1465-1466.

[4] C.X. Liu, T. Liu, L. Liu, K. Liu, A new chaotic attractor, Chaos Solitons Fractals 22 (2004), 1031-1038.

[5] E. Rössler, An equation for continuous chaos, Phys. Lett. A 57 (1976), 397-398.

[6] T. Rikitake, Oscillations of a system of disk dynamos, Proc. R. Cambridge Philos. Soc. 54 (1958), 89-105.

[7] J.H. Lü, G.R. Chen, A new chaotic attractor coined, Int. J. Bifur. Chaos 12 (2002); 659-661. 
[8] F.T. Arecchi, Chaos and generalized multistability in quantum optics, Physica Scripta 9 (1985), 85-92.

[9] A. Algaba, E. Freire, E. Gamero, A.J. Rodríguez-Luis, An exact homoclinic orbit and its connection with the Rössler system, Phys. Lett. A 379 (2015), 1114-1121.

[10] R. Genesio, A. Tesi, Harmonic balance methods for analysis of chaotic dynamics in nonlinear systems, Automatica, 28 (1992), 531-548.

[11] D. Michelson, Steady solutions of the Kuramoto-Sivashinsky equation, Physica D: Nonlinear Phenomena, 19 (1986), 89-111.

[12] J. Llibre, X. Zhang, On the Hopf-zero bifurcation of the Michelson system, Nonlinear Anal. Real World Appl. 12 (2011), 1650-1653.

[13] J. Llibre A. Makhlouf, Zero-Hopf bifurcation in the generalized Michelson system, Chaos Solitons Fractals 89 (2016), 228-231.

[14] Z. Elhadj, J.C. Sprott, Boundedness of certain forms of jerky dynamics, Qual. Theory Dyn. Syst. 11 (2012), 199-213.

[15] X. Wang, G.R. Chen, Constructing a chaotic system with any number of equilibria, Nonlinear Dyn. 71 (2013), 429-436.

[16] Z.C. Wei, W. Zhang, M.H. Yao, On the periodic orbit bifurcating from one single non-hyperbolic equilibrium in a chaotic jerk system, Nonlinear Dyn. 82 (2015), 1251-1258.

[17] Z.C. Wei, J.C. Sprott, H. Chen, Elementary quadratic chaotic flows with a single non-hyperbolic equilibrium, Phys. Lett. A 379 (2015), 2184-2187.

[18] M. Molaie, S. Jafari, J.S. Sprott, S.M. Golpayegani, Simple chaotic flows with one stable equilibrium, Int. J. Bifur. Chaos 23 (2013), 1350188.

[19] J.C. Sprott, Simplest dissipative chaotic flow, Phys. Lett. A 228 (1997), 271-274.

[20] J. Scheurle, J. Marsden, Bifurcation to quasi-periodic tori in the interaction of steady state and Hopf bifurcations, SIAM. J. Math. Anal. 15 (1984), 1055-1074.

[21] I. Baldomá, S. Ibáñez, T.M. Seara, Hopf-Zero singularities truly unfold chaos, Commun. Nonlinear Sci. Numer. Simul. 84 (2020), 105162.

[22] M.R. Cândido, J. Llibre, Zero-Hopf bifurcations in 3-dimensional differential systems with no equilibria, Math. Comput. Simul. 151 (2018), 54-76.

[23] J. Llibre, A. Makhlouf, Zero-Hopf periodic orbit of a quadratic system of differential equations obtained from a third-order differential equation, Differ. Equ. Dyn. Syst. 27 (2019), 75-82.

[24] Y.A. Kuznetsov,t Elements of Applied Bifurcation Theory, Applied Mathematical Sciences, Vol. 112, Springer-Verlag, New York, 1998.

[25] J.A. Sanders, F. Verhulst, J. Murdockm Averaging Methods in Nonlinear Dynamical Systems, Applied Mathematical Sciences, Vol. 59, Springer, New York, 2007.

[26] F. Verhulstm Nonlinear Differential Equations and Dynamical Systems, Universitext, Springer, Berlin, 1990.

[27] A. Buică, J. Llibre, Averaging methods for finding periodic orbits via Brouwer degree, Bull. Sci. Math. 128 (2004), 7-22.

[28] J. Giné, M. Grau, J. Llibre, Averaging theory at any order for computing periodic orbits, Physica D 250 (2013), 58-65.

[29] Hartono, A.H.P. van der Burgh, Higher-order averaging: periodic solutions, linear systems and an application, Nonlinear Anal. 52 (2003), 1727-1744.

[30] R. Eichhorn, S.J. Linz, P. Hänggi, Transformations of nonlinear dynamical systems to jerky motion and its application to minimal chaotic flows, Physical Review E 58 (1998), 7151-7164.

[31] R. Eichhorn, S.J. Linz, P. Hänggi, Simple polynomial classes of chaotic jerky dynamics, Chaos Solitons Fractals 13 (2002), 1-15.

[32] J.C. Sprott, Chaos and Time-Series Analysis, Oxford University Press, Oxford, 2003. 